Has advice been given about the availability of preconception counselling should they plan a pregnancy in future?

Result. On discharge, a total of 33 women were taking one or more antipsychotics and 14 were prescribed a benzodiazepine. 24 women were discharged with antidepressants and 10 women were using a mood stabilising agent. 8 women were discharged without any psychotropic medication.

Overall, 4 women received advice about contraception, and a further 8 women were already using contraception. The impact of taking an antipsychotic on fertility was not discussed with any patient. No women were advised about pre-conception counselling. The impact of taking psychotropic medication on a future pregnancy was discussed with one woman.

Conclusion. Current practice falls well below the standard set by NICE. Opportunities to inform women are being missed, and this has implications for the wellbeing of the patient and, potentially, future children.

Action plan;

Present findings at teaching.

Deliver local teaching covering preconception counselling and the role of adult mental health teams when managing women of childbearing age.

Produce a poster for inpatients wards and an information leaflet for women of childbearing age to aid with discussions.

Create a poster for doctors' offices to remind about NICE standards and documentation.

Re-audit in 6 months.

\section{Audit of patients absent without leave from a psychiatric intensive care unit}

Hamish Naismith*, Mehtab Rahman and Biganani Magadlela

Central and North West London NHS Foundation Trust

${ }^{*}$ Corresponding author.

doi: 10.1192/bjo.2021.283

Aims. We aimed to reduce the number of patients absent without leave (AWOL) by carrying out an audit of processes around granting leave for those patients and the action taken when they absconded. We also wanted to determine factors which might be associated with patients absconding.

Background. Nile ward is a 14-bedded male psychiatric intensive care unit (PICU). All patients admitted to the ward are under section 2 or 3 of the Mental Health Act. Patients who are AWOL may pose a risk of harm to themselves or others. The Royal College of Psychiatrists' Quality Network for PICUs has developed applicable standards, which include criteria on developing a leave plan, actions to take when patients are AWOL and involvement of carers.

Method. Patients who went AWOL during a six month period in 2019 from ward records. The electronic medical records for identified patients were reviewed to assess whether the following eight criteria were met: risk assessment documented; leave conditions specified; consultation with the multi-disciplinary team; crisis card provided to patients or families; risk management plan enacted when AWOL; relevant authorities informed; incident form completed; relatives/carers involved in patient's care if they consented. In reviewing the notes, factors that might have been associated with an increased risk of AWOL were also assessed in order to inform risk assessment.

Result. Six patients were identified who went AWOL during the six month period in question. For six of the criteria, all of patients' cases met the audit standards. Five patients' did have involvement of relaties/carers if they consented, but in one case no details were available for making contact. All patients lacked documented details of crisis numbers being provided before they went on leave. Preliminary findings that might be associated with an increased risk of AWOL are differing views between the patient and the treating team on the care plan and concerns about mental state.

Conclusion. The audit showed many of the standards are met. However, a quality improvement intervention is planned to ensure all audit standards are met, in particular around providing a crisis card to patients and these findings will be presented on the poster, if accepted. Further research is needed into factors which might be associated with an increased risk of absconsion in PICU.

\section{The effectiveness of community treatment orders (CTOS) across Birmingham and Solihull Mental Health Foundation Trust (BSMHFT)}

Sambavi Navaratnarajah ${ }^{1 \star}$, Riya Basu ${ }^{2}$ and Syed Rashid ${ }^{1}$

${ }^{1}$ BSMHFT and ${ }^{2}$ North AOT, BSMHFT

${ }^{*}$ Corresponding author.

doi: 10.1192/bjo.2021.284

Aims. To ascertain if CTOs ensure that patients are effectively treated in the community and maintain stability in their mental health.

If a patient requires recall(+/- revocation) that this is done in a timely fashion in according to the 1983 Mental Health Act.

Background. A number of studies have been inconclusive in determining the benefits of CTOs in reducing the re-admissions of "revolving door" patients In Assertive Outreach (AO). It is felt that CTOs have reduced readmission of patients due to the intensive input from community teams, decreased recall and subsequent revocation. Those admitted are thought to require fewer inpatient days. It is clear that many patients who require recall following non-engagement, non-compliance, will accept medication following RC review. However at present in BSMHFT patients can only be recalled if they are allocated a bed. Due to the national bed shortage, this has resulted in delays following decision to recall and thus early and effective treatment for patients. In this aspect it defeats the role of the CTO as per the 2007 MHA.

Method. CTO data from 1st April 2018 to 31st March 2019 was obtained from all 6 AOT's in BSMHFT. The following factors were considered;

1. Time between decision to recall by $\mathrm{RC}$ and recall to inpatient facility

2. Number of recalls converted to revocations

3. Number of inpatient bed days if revoked

4. Number of admissions on CTO

5. Patient/family agreement of CTO

Result. 98 CTO patients were recorded over this period. 19 out of 26 recalls had recall dates documented. 10 recalls were revoked due to relapse of mental illness. Average days from RC recall decision to actual recall or cancellation was 63.89 days. Main reasons for delay were bed unavailability and execution of warrant.

Following revocation, average inpatient bed days was 103.71 . $41 \%$ of families agreed with CTOs, $36 \%$ of patients contested their CTO.

Conclusion. Over a quarter of patients on CTO were recalled to hospital however, less than half of these had their CTO revoked. The remainder accepted treatment following urgent community review whilst on the bed list. Evidently the majority of patients didn't need admission. With the ongoing bed crisis, alternative 
avenues need to be sought to ensure prompt treatment and prevent relapse.

A CTO suite designed for recall could be the solution for the future.

\section{Overdose admissions to a district general hospital intensive care unit}

Aoife Nechowska*, Theophilus Samuels and Sameer Ranjan

Surrey and Sussex Healthcare NHS Trust

${ }^{\star}$ Corresponding author.

doi: 10.1192/bjo.2021.285

Aims. This audit aimed to analyse the patient population coming into East Surrey Hospital's Intensive Care Unit from 1993 to 2019. Background. The Office for National Statistics (ONS) published a report in August 2019 on ages most likely to die by suicide and drug poisoning. Their data showed that Generation $\mathrm{X}$ were dying by this method in greater numbers than other age groups. This is in contrast with data from 1990s for England and Wales which showed people in their 20s were most likely to die by suicide or poisoning. This audit set out to look at admission data from an intensive care unit (ICU) in a district general hospital in Surrey over a similar period of time.

Method. Patient records from 1993 to September 2019 were accessed using the WardWatcher database. To access the maxim number of admissions qualifying under the aims, the database was accessed by searching under "admission comments" for: overdose, self-harm, poison, suicide. These reports were downloaded and the lists were checked against each other to delete duplicates. This gave a total of 331 patients. The data were analysed by year, according to age, gender, season, psychiatric diagnoses and previous overdose attempts. Their outcomes were checked against recorded deaths. There was not enough information to investigate method of overdose.

Result. A total of 331 patient records were accessed. The youngest patient was 15 years old, the oldest was 84 years old. The age dataset was non-parametrically distributed with the median age of 43 years (IQR 33-51 years). The age distributions for each year appeared symmetrical but total numbers for each year were small. The population was split as 191 female (58\%) and 141 male (42\%). 16 patients died on the ICU on admission with an overdose, $5 \%$ of total numbers, of which $19 \%$ had a previous overdose attempts and $44 \%$ had a psychiatric diagnosis. The youngest death was 22 and the oldest was 81 years old. The average age was 47 years, with the spread consistent in the 2000s and 2010s.

Conclusion. The results from East Surrey Hospital's ICU do not reflect the analysis from the ONS. The mean age for each year has remained similar. Numbers for the audit were small and admission criteria to the ICU prescribe that the patient be critically unwell and may not be indicative of the total admissions to a district general hospital.

\section{Neuroimaging in an older adult inpatient psychiatric unit}

Aoife Nechowska

Surrey and Borders Partnership NHS Foundation Trust

doi: 10.1192/bjo.2021.286

Aims. This audit aimed to analyse the use of neuroimaging and its effect on treatment in an older adult inpatient psychiatry unit over the period of one year.
Background. Brain imaging can be used as a diagnostic tool in psychiatry. According to NICE guidelines, structural imaging, such as magnetic resonance imaging (MRI) or computed topography (CT), can be used in the workup for dementia diagnosis in order to exclude non-dementia pathology and identify dementia subtype. This is important in the geriatric population where evidence of small vessel disease has an impact on treatment options and management of polypharmacy.

Method. A list of patients from the past year (January to December 2019) was accessed. Patient records were then analysed to see if neuroimaging had been accessed during their admission at The Meadows Hospital, Surrey and Borders Partnership. This included imaging from prior to admission. Analysis was divided into type of imaging, comments and impact on diagnosis.

Result. Overall numbers for the audit were small. A total of 74 patients were admitted onto the unit, of which 3 were readmissions. There was missing information for 8 patients, giving a total of 63 patients. CT scans were accessed for 35 patients ( $56 \%$ of total); 3 of these were done during the admission. MRI scans were completed for 21 patients (33\%), with one requested during admission. A total of 9 patients (14\%) had both CT and MRI scans. Neuroimaging results led to a change in diagnosis for 6 patients (10\%). In all cases this reflected the finding of small vessel disease and a change of diagnosis to either vascular dementia or mixed dementia.

Diagnoses were also analysed. The Meadows Hospital has 2 dementia wards (male and female) and 1 functional ward (for females). A total of 36 patients (57\%) were diagnosed with dementia, of which the biggest groups were: Alzheimer's dementia (13 patients, $36 \%$ ) and Vascular dementia (11 patients, $31 \%$ ). Conclusion. The majority of the patients were admitted with established diagnoses and so only a small number had changes made following review of imaging. Good imaging results and reports help to differentiate types of dementia. Although neuroimaging is not gold standard in diagnosing dementia syndromes, it is now an important aspect in the diagnostic pathway. Getting the diagnosis correct will help with treating individuals appropriately and avoid unnecessary prescribing.

\section{Implementation of smoking cessation policy at the} antelope house in Southern Health NHS Foudation Trust in Southampton

Sunday Neru* and Edet Ededet

Antelope House, Southern Health NHS Foundation Trust, Brintons Terrace, Southampton

${ }^{\star}$ Corresponding author.

doi: 10.1192/bjo.2021.287

Aims. To ensure that health care practitioners at Antelope house, Southern Health NHS Foundation Trust in Southampton are providing service users information, advice and stop smoking support in line with smoke free policies in the trust.

Background. Smoking is the single largest preventable cause of ill health and premature death in England. Cigarette smoking causes a wide range of diseases and medical conditions like cancers, heart diseases and stroke.

The prevalence of stroke is extremely high among people with mental health problems especially those admitted to hospital.

Stopping smoking reduces the risk of developing preventable diseases and premture death.

These are the background behind this audit. 